\title{
Potential players in the hood
}

\author{
Penelope A. Boyden • Richard B. Robinson
}

Received: 16 May 2012 / Accepted: 22 May 2012 /Published online: 26 July 2012

(C) Springer Science+Business Media, LLC 2012

Pharmacologic treatment of cardiac arrhythmias has been mostly confined to drugs that alter the electrical function of the myocytes of the heart. In fact, big players in this market have been the adrenergic blocking agents. By virtue of modulation of autonomic tone, this class of drugs can be helpful. However in the case of atrial fibrillation (Af), most current agents are not that helpful and the course of Af is little changed. It is possible that other players in the neighbor "hood" of cells may gain importance.

In the paper of Mao et al. [1], autonomic modulation takes on an additional meaning in that in this study, it is the modulation of atrial neural networks, namely those of the fat pads, that these authors believe promote Af. This is consistent with their ideas that autonomic nerve stimulation or autonomic denervation modulates autonomic activity in physiologic and/or pathophysiologic functions. Notably, the experiments of Mao et al. were completed using naïve control animals and not animals susceptible to persistent Af. In fact, the longest Af duration noted was $33 \mathrm{~min}$ in the presence of acetylcholine (Ach), which was used to mimic the neurotransmitter released with stimulation of the left and right ganglionated plexi (GPs). It is not known whether the same outcomes would occur in animals with

This study was supported by grant HL 66140 from the National Heart Lung and Blood Institute, Bethesda, MD, USA.

P. A. Boyden · R. B. Robinson

Department of Pharmacology,

Center for Molecular Therapeutics, Columbia University,

New York, NY 10032, USA

P. A. Boyden ( $\square)$

Department of Pharmacology,

Columbia College of Physicians and Surgeons,

630 West 168 th ST,

New York, NY 10032, USA

e-mail: pab4@columbia.edu highly remodeled or even aged atria as well as if remodeled GPs had been used.

The mechanism of remodeling of the Af substrate by atrial neural networks was not explored in this study and thus remains unknown. We know that disease-associated disruptions of adrenergic/cholinergic signaling would be a form of functional denervation and if these paths serve to maintain the adult cardiac phenotype (and if functional and anatomic denervation have equivalent effects) then that could lead to additional myocyte remodeling. There are clear examples in the literature that Af produced by rapid atrial pacing, and VT, $\mathrm{Vf}$ and sudden death are associated with heterogeneous atrial innervation in the form of nerve sprouting [2].

Atrial cell electrical [3-6] or autonomic remodeling [7] may be just a reflection of an altered antagonism between the parasympathetic and sympathetic controls of the atria. It is known that vagal input can inhibit sympathetic neurotransmission via (Ach) receptors on the sympathetic nerve terminals. Sympathetic stimulation can produce long-lived vagal inhibition. This latter effect is thought to be caused by neuropeptideY (NPY) which colocalizes with Norepinephrine in sympathetic nerve terminals.

NPY effects are varied showing both acute and chronic effects on cardiac cells. Using myocardial neuronal cocultures, labs have now defined some aspects of how neurons first impact the cardiac phenoptype [8-10]. One of us has studied intensely how sustained sympathetic innervation regulates ion channel expression and autonomic responsiveness and how some of these effects can be ascribed to NPY acting as a trophic factor [11, 12].

Mao et al. suggest that another peptide, VIP, may play a role. VIP effects are less well defined. It is released with Ach but causes a marked increase in HR. Whether it too has trophic effects is not known. Obviously more needs to be done here using cardiac tissues. 
Thus, ablation of nerves (in the Mao et al. paper) appears to be a way of suppression of certain types of arrhythmias, but the effects of such on other players in the hood should be considered since their post ablation remodeling may exacerbate the arrhythmia substrate.

\section{References}

1. Mao, J., Scherlag, B. J., Liu, Y., Fan, Y., Varma, V., Stavrakis, S., $\&$ Po, S. (2012) The atrial neural network as a substrate for atrial fibrillation. Journal of Interventional Cardiac Electrophysiology. doi:10.1007/s10840-012-9692-3

2. Shen, M. J., Choi, E. K., Tan, A. Y., Lin, S. F., Fishbein, M. C., Chen, L. S., et al. (2012). Neural mechanisms of atrial arrhythmias. Nature Reviews Cardiology, 9, 30-39.

3. Yagi, T., Pu, J., Chandra, P., Hara, M., Danilo, P. D., Jr., Rosen, M. R., \& Boyden, P. A. (2002). Density and function of inward currents in right atrial free wall cells from chronically fibrillating canine atria. Cardiovascular Research, 54, 405-415.

4. Hara, M., Shvilkin, A., Rosen, M. R., Danilo, P. D., Jr., \& Boyden, P. A. (1999). Steady state and nonsteady state action potentials in fibrillating canine atrium: abnormal rate adaptation and its possible mechanisms. Cardiovascular Research, 42, 455-469.
5. Dun, W., Chandra, P., Danilo, P., Rosen, M. R., \& Boyden, P. A. (2003). Chronic atrial fibrillation does not further decrease outward currents. It increases them. American Journal of Physiology, 285, H1378-H1384.

6. Dun, W., \& Boyden, P. (2009). Aged atria: electrical remodeling conducive to atrial fibrillation. Journal of Interventional Cardiac Electrophysiology, 1(25), 9-18.

7. Yu, L., Scherlag, B. J., Sha, Y., Li, S., Sharma, T., Nakagawa, H., et al. (2012). Interactions between atrial electrical remodeling and autonomic remodeling: how to break the vicious cycle. Heart Rhythm, 9, 804-809.

8. Drugge, E. D., Rosen, M. R., \& Robinson, R. B. (1985). Neuronal regulation of the development of the alpha-adrenergic chronotropic response in the rat heart. Circulation Research, 57, 415-423.

9. Zhang, J. F., Robinson, R. B., \& Siegelbaum, S. (1992). Sympathetic neurons mediate developmental change in cardiac sodium channel gating through long-term neurotransmitter action. Neuron, 9, 97-103.

10. Protas, L., Barbuti, A., Qu, J., Rybin, V. O., Palmiter, R. D., Steinberg, S. F., et al. (2003). Neuropeptide Y is an essential in vivo developmental regulator of cardiac ICa,L. Circulation Research, 14(93), 972-979.

11. Protas, L., Qu, J., \& Robinson, R. B. (2003). Neuropeptide Y: neurotransmitter or trophic factor in the heart? Physiology, 1(18), 181-185.

12. Protas, L., \& Robinson, R. B. (2008). Dissecting the NPY signaling cascade between cardiac sympathetic and parasympathetic nerves. Journal of Molecular and Cellular Cardiology, 44, 470-472. 